\title{
Aberrant brain functional connectome in patients with obstructive sleep apnea
}

This article was published in the following Dove Press journal:

Neuropsychiatric Disease and Treatment

\section{Li-Ting Chen $1, *$ \\ Xiao-Le Fan ${ }^{2, *}$ \\ Hai-Jun Li' \\ Cheng-Long Ye' \\ Hong-Hui Yu' \\ Hui-Zhen Xin' \\ Hong-Han Gong' \\ De-Chang Peng' \\ Li-Ping Yan ${ }^{3}$}

'Department of Radiology, the First Affiliated Hospital of Nanchang University, Nanchang, Jiangxi Province,

China; ${ }^{2}$ Department of General

Surgery, the First Affiliated Hospital of Nanchang University, Nanchang, Jiangxi Province, China; ${ }^{3}$ Department of Cardiology, People's Hospital of Jiangxi Province, Nanchang, Jiangxi Province, China

*These authors contributed equally to this work
Correspondence: De-Chang Peng

Department of Radiology, the First Affiliated Hospital of Nanchang

University, No 17, Yongwai Zheng Street, Donghu District, Nanchang 330006,

Jiangxi Province, China

Tel +86 79l 88694457

Email pengdcdoctor@I63.com

Li-Ping Yan

Department of Cardiology, People's Hospital of Jiangxi Province, No 92, Aiguo Street, Donghu District, Nanchang 330006, Jiangxi Province, China

Tel +86 79l 86892I80

Email 824852148@qq.com
Objective: Obstructive sleep apnea (OSA) is accompanied by widespread abnormal spontaneous regional activity related to cognitive deficits. However, little is known about the topological properties of the functional brain connectome of patients with OSA. This study aimed to use the graph theory approaches to investigate the topological properties and functional connectivity (FC) of the functional connectome in patients with OSA, based on resting-state functional magnetic resonance imaging (rs-fMRI).

Methods: Forty-five male patients with newly diagnosed untreated severe OSA and 45 male good sleepers (GSs) underwent a polysomnography (PSG), clinical evaluations, and rs-fMRI scans. The automated anatomical labeling (AAL) atlas was used to construct the functional brain connectome. The topological organization and FC of brain functional networks in patients with OSA were characterized using graph theory methods and investigated the relationship between functional network topology and clinical variables.

Results: Both the patients with OSA and the GSs exhibited high-efficiency "small-world" network attributes. However, the patients with OSA exhibited decreased $\sigma, \gamma, \mathrm{E}_{\text {glob }}$; increased Lp, $\lambda$; and abnormal nodal centralities in several default-mode network (DMN), salience network (SN), and central executive network $(\mathrm{CEN})$ regions. However, the patients with OSA exhibited abnormal functional connections between the DMN, SN, and CEN. The disrupted FC was significantly positive correlations with the global network metrics $\gamma$ and $\sigma$. The global network metrics were significantly correlated with the Epworth Sleepiness Scale (ESS) score, Montreal Cognitive Assessment (MoCA) score, and oxygen desaturation index.

Conclusion: The findings suggest that the functional connectome of patients with OSA exhibited disrupted functional integration and segregation, and functional disconnections of the DMN, $\mathrm{SN}$, and CEN. The aberrant topological attributes may be associated with disrupted FC and cognitive functions. These topological abnormalities and disconnections might be potential biomarkers of cognitive impairments in patients with OSA.

Keywords: obstructive sleep apnea, graph theory, functional connectome, cognitive function, resting-state fMRI

\section{Introduction}

Obstructive sleep apnea (OSA) is a common sleep-related breathing disorder characterized by repetitive partial (hypopneas) or complete (apnea) obstruction of the upper airway during sleep, which leads to fragmented sleep, intermittent nocturnal hypoxia, and hypercapnic hypoxia. ${ }^{1}$ Population-based epidemiological studies indicate that OSA is estimated to occur in $12 \%$ of women and up to $30 \%$ of men aged between 30 and 70 years, and the prevalence rates are increasing with increasing population age and obesity levels. ${ }^{2}$ OSA is associated with a broad range of neurocognitive impairment, psychological problems, cardiovascular and cerebrovascular diseases, insomnia, 
and even Alzheimer's disease. The detrimental effects of OSA include impaired work performance, daytime sleepiness, decreased quality of life, increased risk of vehicular and industrial accidents, and cognitive deficits. Patients with OSA exhibit neurocognitive impairments that involve attention, memory, learning, executive function, visuospatial function, and language abilities. Sleep fragmentation and intermittent nocturnal hypoxia are presumed to be the main contributory factors in neuropsychological impairments in patients with OSA; ${ }^{1,3}$ however, the underlying neuropathological mechanisms of cognitive impairments in patients with OSA remain largely unclear. To explain cognitive deficits, magnetic resonance imaging (MRI), a non-invasive imaging technique, has been widely used to identify brain structural and functional impairments in OSA patients. Several studies have suggested that widespread local abnormalities in OSA, expressed as reduced white matter integrity and gray matter volume, brain metabolites abnormalities, ${ }^{4}$ aberrant regional cerebral blood flow, ${ }^{5}$ and altered brain activation and deactivation patterns in multiple brain regions, ${ }^{6-9}$ particularly in the default-mode network (DMN), salience network (SN), and central executive network (CEN), are responses for the evoked sensorimotor, affective, autonomic, and ventilatory challenges and cognitive deficits. ${ }^{10-12}$

Resting-state functional MRI (rs-fMRI) is a promising tool for understanding neural deficits, monitoring disease progression, and evaluating treatment effects in patients with OSA, ${ }^{13}$ and rs-fMRI has been widely used to explore the intrinsic brain activity of humans. ${ }^{14}$ Previous rs-fMRI studies have suggested that OSA specifically affects resting-state brain activity in several regions, including the insular cortex, hippocampus, posterior cingulate cortex (PCC), thalamus, basal ganglia, frontal regions, cerebellum, and sensorimotorrelated areas. ${ }^{8,9}$ Functional connectivity (FC) alterations have been identified between specific region pairs in patients with OSA. Zhang et $\mathrm{al}^{15}$ found a functional disconnection between the right anterior insula (AIns) and the DMN that was associated with OSA severity and impaired working memory performance; the authors regard the weakened rs-FC of the right AIns with the DMN to be a biomarker of cognitive impairment in patients with OSA. OSA selectively impairs the rs-FC of subregions within the DMN, which is associated with cognitive impairments. ${ }^{6}$ In addition, structural and functional deficits in the anterior DMN, but functional compensation in the posterior DMN, have been found in patients with OSA. ${ }^{13}$ Park et al ${ }^{16}$ observed abnormal rs-FC in various brain regions, including autonomic, affective, executive, and sensorimotor functional areas related to cognitive functions in patients with OSA. Despite the increasing knowledge of impaired spontaneous regional activity and abnormal FC, either within a specific brain system or between different systems of OSA, ${ }^{7-9}$ very little is known regarding whether OSA disrupts the global topological organization of the brain functional connectome or the relationship between FC and global topological organization.

Graph theoretical analysis provides a powerful framework for characterizing the topological properties of brain networks, including global and regional properties, and has become increasingly popular in the neuroimaging field. Through graph theory approaches, the functional network of the human brain has been found to show prominent smallworld attributes that are simultaneously highly segregated and integrated, and are characterized by a high degree of local interconnectivity and small path lengths linking nodes in individual networks. ${ }^{17}$ Graph theory approaches have been applied to study not only normal brains ${ }^{18}$ but also various brain-related diseases, such as depression, ${ }^{19}$ Alzheimer's disease, ${ }^{20}$ posttraumatic stress disorder, ${ }^{21}$ schizophrenia, ${ }^{22}$ and epilepsy. ${ }^{23}$ An increasing number of studies have used graph theory and combined FC with the small-world network organization of brain functions to investigate the effects of diseases on brain networks and have shown that cognitive deficits are accompanied by disrupted topological organization. ${ }^{24}$ The relationship between functional network topology and cognitive function in OSA is an important question that has so far been relatively under-explored, although current work has shown variations in the topological properties of functional and structural networks in OSA. ${ }^{25,26}$

We hypothesized that the topological organization of the brain functional connectome has been disrupted in patients with OSA and that these alterations may be related to the disrupted rs-FC and cognitive impairments of patients with OSA. Here, we used graph theory approaches and rs-fMRI to investigate changes in the topological organization of functional networks in OSA patients. In this study, we constructed the functional connectome of OSA and sought to determine whether patients with OSA would show the following: (1) abnormal global and regional properties in brain functional networks; (2) disrupted functional network connectivity; and (3) the relationship between network topological property changes and FC, clinical variables, and cognitive performance.

\section{Methods}

\section{Subjects}

Forty-five male patients with untreated severe OSA who were recruited from the Sleep Monitoring Room of the 
First Affiliated Hospital of Nanchang University, China, and 45 demographically matched good sleepers (GSs) from the local community participated in this study, between October 2015 and August 2017. The following inclusion criteria were used for the OSA patients and GSs in this study: (1) OSA patients, an apnea-hypopnea index (AHI) greater than or equal to 30 ; GSs, as healthy control, with an AHI less than 5; (2) right-handed; (3) male gender; and (4) age older than 20 years but younger than 60 years. The following exclusion criteria for the OSA patients and GSs were employed: (1) other sleep disorders, such as primary insomnia or a sleep-related eating disorder; (2) a structural lesion in conventional MR images; (3) neurological or mental illnesses (eg, neurodegenerative diseases, hypothyroidism, epilepsy, depression, psychosis, and head injury); (4) addiction to drugs or alcohol; (5) chronic obstructive pulmonary disease or diabetes cerebrovascular disease; (6) MRI contraindications, such as claustrophobia, metallic implants, or devices in the body; and (7) refusal by the participant to enroll in this study. This study was approved by the Institutional Review Board of the First Affiliated Hospital, Nanchang University, China. This study was conducted in compliance with the principles of the Declaration of Helsinki. Written informed consent was provided by all participants before beginning any study procedure.

\section{Polysomnography (PSG)}

Full nocturnal PSG was performed on patients with OSA and GSs using the Respironics LE-Series Physiological Monitoring System (Alice 5 LE; Respironics, Orlando, FL, USA). One day prior to the overnight PSG, all participants were required to refrain from consuming hypnotics, alcoholic drinks, and coffee. Overnight PSG was recorded from $10 \mathrm{PM}$ to $6 \mathrm{AM}$ the next morning. Standard electroencephalogram (EEG), electrooculogram (EOG), chin electromyogram (EMG), electrocardiogram (ECG), thoracic and abdominal respiratory movements, oral and nasal airflow, oxygen saturation $\left(\mathrm{SaO}_{2}\right)$, snoring, and body position were recorded. According to the American Academy of Sleep Medicine guidelines, obstructive apnea was defined as a reduction in airflow $\geq 90 \%$ lasting for more than $10 \mathrm{~s}$ and associated with persistent respiratory effort; a hypopnea was defined as a reduction in airflow $\geq 30 \%$ lasting at least $10 \mathrm{~s}$ and accompanied by a $4 \%$ or greater oxygen desaturation and/or with EEG arousal. ${ }^{27}$ The AHI was calculated as the average number of apnea and hypopnea events experienced per hour during sleep. The arousal index (AI) was calculated as the mean number of EEG arousals per hour of sleep.

\section{Neuropsychological assessments}

Excessive daytime sleepiness was evaluated by using the Epworth Sleepiness Scale (ESS, Chinese version), which requires participants to rate his or her probability of falling asleep on a scale of increasing probability from 0 to 3 in eight different situations. ${ }^{28}$ The maximum score is 24 . A score greater than 6 suggests sleepiness, a score greater than 11 demonstrates excessive sleepiness, and a score greater than 16 indicates risky sleepiness. Cognitive function was evaluated using the Montreal Cognitive Assessment (MoCA, Chinese version) ${ }^{29}$ with eight cognitive items, including executive function, memory, attention, calculation, abstraction, naming, language, and orientation. The maximum MoCA score is 30, with a MoCA score less than 26 indicating cognitive impairment. If the number of schooling years was less than 12 , one point was added to adjust for educational deviations. $^{30}$

\section{MRI data acquisition}

All MRI data were collected on a 3.0 T MRI system (Siemens, Erlangen, Germany) scanner using an 8-channel phased-array head coil in the First Affiliated Hospital of Nanchang University, China. Foam pads were used to restrict head movements, and ear plugs were used to minimize scanner noise. Participants were trained how to swallow, keep nasal breathing, and, without open mouth, to control the movement of the tongue and the mandible. All the participants were required to remain as still as possible, to relax, to keep their eyes closed, to not think systematically, and to not fall asleep. First, conventional T1-weighted images and T2-weighted images were collected for all the participants to exclude macrostructural brain lesions that may affect brain function or microstructure. Resting-state functional images with a gradient-recalled echoplanar imaging (EPI) sequence (repetition time $=2,000 \mathrm{~ms}$, echo time $=30 \mathrm{~ms}$, thickness $=4.0 \mathrm{~mm}$, gap $=1.2 \mathrm{~mm}$, field of view $=230 \times 230 \mathrm{~mm}$, flip angle $=90^{\circ}$, matrix $=64 \times 64$, slices $=30$, duration $8 \mathrm{~min}$ and $6 \mathrm{~s}, 30$ axial slices covering the whole brain) were obtained. Finally, high-resolution threedimensional T1-weighted brain structural MR images were obtained using a magnetization-prepared rapid gradient echo sequence (repetition time $=1,900 \mathrm{~ms}$, echo time $=2.26 \mathrm{~ms}$, thickness $=1.0 \mathrm{~mm}$, gap $=0.5 \mathrm{~mm}$, field of view $=250 \times 250 \mathrm{~mm}$, flip angle $=9^{\circ}$, resolution matrix $=256 \times 256$, slices $=176$ ). After the MRI scan, all the participants were asked whether they fell asleep during the scan.

\section{fMRI data preprocessing}

Data were preprocessed using Statistical Parametric Mapping (SPM8) (http://www.fil.ion.ucl.ac.uk) and Data 
Processing \& Analysis Assistant for Resting-State Brain Imaging (DPABI, Chinese Academy of Sciences, Beijing, China, http://rfmri.org/DPABI $)^{31}$ in the MATLAB 2012a (MathWorks, Natick, MA, USA) platform. The first 10 volumes of each subject were discarded to eliminate magnetic saturation effects, and the remaining 230 volumes were slice-time corrected and three-dimensional head motion corrected. Subjects were excluded if there was more than 1.5-mm maximum displacement in any of the directions ( $\mathrm{x}, \mathrm{y}$, $\mathrm{z}$ ), or more than $1.5^{\circ}$ of angular rotation in any axis during the whole fMRI scans, or if there was a frame-wise displacement (FD) of more than 2.5 standard deviations $^{32}$ for any of the 230 volumes. The T1-weighted structural images were coregistered to the mean of the realigned EPI images for each individual. The transformed structural images were then segmented into gray matter, white matter, and cerebrospinal fluid using the new segmentation in SPM8. The Diffeomorphic Anatomical Registration Through Exponentiated Lie Algebra tool was used to compute transformations from individual native space to Montreal Neurological Institute (MNI) space, and images were re-sampled to $3 \times 3 \times 3 \mathrm{~mm}^{3}$ voxels. Then, the images were smoothed with a $6-\mathrm{mm}$ full-width at halfmaximum Gaussian kernel. To further reduce the effects of confounding factors, nuisance covariates, including the white matter signal, cerebrospinal fluid signal, global signal, and Friston 24-parameter head motion parameters, were regressed from the time series of all voxels via linear regression. ${ }^{33}$ Finally, a temporal bandpass filtering $(0.01-0.08 \mathrm{~Hz})$ was performed to reduce low-frequency drift, physiological high-frequency noise, and cardiac noise.

\section{Functional network construction Node and edge definitions}

At the microscopic scale, a human brain network can be described abstractly by a graph or subgraph, with nodes defining neurons and edges representing axonal connections. However, at the macroscopic scale of neuroimaging data, the nodes represent voxels or regions, and the edges are some statistical measure of association (ie, FC) between voxels or regions. To define the brain nodes, automated anatomical labeling (AAL) was employed, which divided the whole brain into 116 cortical and subcortical regions of interest. ${ }^{34,35}$ For each subject, the averaged time series of all voxels in each region of interest was extracted to obtain a representative time series. The Pearson's correlation coefficients between the regional mean time series of all possible pairs of the 116 brain regions were calculated as edges in the network, resulting in a $116 \times 116$ Pearson's correlation matrix for each participant, and then converted into binarized matrices. A Fisher's r-to-z transformation was performed to translate the individual correlation maps into z-scored maps to improve normality. ${ }^{36}$

\section{Network analysis}

In this study, both the global and regional network measures of functional brain networks of patients with OSA and GSs were investigated using the graph theoretical network analysis toolbox (GRETNA) (http://www.nitrc.org/projects/ gretna/). ${ }^{37}$ The brain functional networks were modeled based on an unweighted, undirected method. Sparsity (Sp) was defined as the ratio of the existing edges, divided by the highest possible number of edges in a network, which ensured that the all resultant networks would have the same number of edges and minimized the effects of possible discrepancies in the overall correlation strength between the groups. ${ }^{19,38}$ Therefore, we applied a Sp threshold to all correlation matrices over a wide range of Sp levels (from 0.05 to 0.40 using intervals of 0.01 ) to explore the between-group differences in the brain functional network organization of patients with OSA and GSs. The area under the curve (AUC) of each network metric was calculated over the Sp range from $\mathrm{S}_{1}$ to $\mathrm{S}_{\mathrm{n}}$, with an interval of $\Delta \mathrm{S}$, and this depicts the change in topological characterization of the brain networks. ${ }^{19}$ The integrated AUC metric is sensitive at detecting topological alterations of brain disorders, and has been used in previous studies. ${ }^{24,39,40}$ The global network metrics examined in the present study included the following: 1) the small-world properties, ${ }^{17}$ clustering coefficient, $\mathrm{Cp}$, characteristic path length, Lp, normalized clustering coefficient, $\gamma$, normalized characteristic path length, $\lambda$, and small-worldness, $\sigma$; and 2 ) the network efficiency parameters, ${ }^{39,41}$ global efficiency, $\mathrm{E}_{\text {glob }}$, and local efficiency, $\mathrm{E}_{\text {loc }}$. A small-world network with a much higher clustering coefficient $(\mathrm{Cp})$ and a similar characteristic path length (Lp) compared with random networks, met the following criteria: normalized clustering coefficients, $\gamma=\mathrm{Cp} \mathrm{p}_{\text {real }} / \mathrm{Cp}$ rand $>1$, and normalized characteristic path length, $\lambda=\mathrm{Lp}_{\text {real }} / \mathrm{Lp}_{\text {rand }} \approx 1$, or the small-worldness equation, $\sigma=\gamma / \lambda>1 .{ }^{17}$ To determine the regional (nodal) characteristics of functional networks, nodal degree, nodal efficiency $\left(\mathrm{E}_{\text {nodal }}\right)$, and nodal betweenness centrality were computed in this study.

\section{Statistical analysis}

Demographic and clinical variables of the OSA patients and GSs were compared using two-sample $t$-tests with IBM Statistical Package for the Social Sciences 20.0 software 
(IBM SPSS Inc., Chicago, IL, USA). To determine the between-group differences in the small-world properties $(\mathrm{Cp}$, Lp, $\gamma, \lambda$, and $\sigma)$, network efficiency $\left(\mathrm{E}_{\text {glob }}, \mathrm{E}_{\text {loc }}\right)$, and nodal characteristics (nodal degree, nodal efficiency, and betweenness centrality), the AUC of each metric was calculated for statistical comparison under the $\mathrm{Sp}$ range $(0.05<\mathrm{Sp}<0.40$, step $=0.01$ ) with age, educational level, and mean FD as covariates. A false discovery rate (FDR) procedure was further performed at a q-value of 0.05 to correct for multiple comparisons. $^{42}$

To localize the specific pairs of brain regions with altered FC in the patients with OSA, we chose the pairs of regions that exhibited between-group differences in at least one of the three nodal centralities, including nodal degree, nodal efficiency, and nodal betweenness, and then utilized the network-based statistics (NBS) approach ${ }^{43}$ (www.nitrc. org/projects/nbs/) to localize the connected networks that showed significant alterations in the patients with OSA. Then, a subset of connection matrices was generated based on these altered nodes in patients with OSA. We applied the NBS method to define a set of suprathreshold links among any connected components (threshold, $\mathrm{T}=2.368, P<0.01$ ). The significance of each component was estimated using the nonparametric permutation method $(10,000$ permutations). Age, educational level, and mean FD were taken as covariates of no interest in this model. Finally, to evaluate the clinical correlates of these network property changes in OSA patients, a partial correlation analysis was performed to assess the relationships between network metrics and clinical variables.

\section{Results}

\section{Demographic and clinical data}

There were no significant differences in age $(P=0.893)$ and education $(P=0.817)$ between the OSA patients and the GSs. There were significant between-group differences in BMI, AHI, nadir $\mathrm{SaO}_{2}$, average $\mathrm{SaO}_{2}, \mathrm{~N} 1, \mathrm{REM}, \mathrm{SaO}_{2}<90$ percentage, AI, oxygen, desaturation index, MoCA score, ESS score, and sleep efficiency between the OSA patients and the GSs (Table 1).

\section{Small-world properties of brain functional networks}

In the defined threshold range (here from 0.05 to 0.40 , step $=0.01$ ), both the patients with OSA and the GSs exhibited robviously larger than $1\left(\gamma_{\mathrm{OSA}}=1.74 \pm 0.80, \gamma_{\mathrm{GSs}}=1.86 \pm 0.90\right)$ and $\lambda$ approximately equal to $1\left(\lambda_{\mathrm{OSA}}=1.08 \pm 0.15, \lambda_{\mathrm{GSs}}=1.06 \pm 0.10\right)$. $\sigma(\sigma=\gamma / \lambda)$ was larger than $1\left(\sigma_{\mathrm{OSA}}=1.59 \pm 0.58, \sigma_{\mathrm{GSs}}=1.73 \pm 0.65\right)$,
Table I Demographic and clinical data comparisons between the patients with OSA and the GSs

\begin{tabular}{|c|c|c|c|c|}
\hline Chare & $\begin{array}{l}\text { Patients } \\
\text { with OSA } \\
(n=45)\end{array}$ & GSs $(n=45)$ & t-value & $P$-value \\
\hline ge (years) & $37.56 \pm 8.86$ & $37.84 \pm 11.38$ & -0.134 & 0.893 \\
\hline ducation (years) & $|2.3| \pm 2.75$ & $|2.18 \pm 2.7|$ & 0.232 & 0.817 \\
\hline BMI $\left(\mathrm{kg} / \mathrm{m}^{2}\right)$ & $27.53 \pm 3.33$ & $23.10 \pm 1.98$ & 7.656 & $<0.00$ I* $^{*}$ \\
\hline $\mathrm{AHI}$ & $58.7 \pm 20.38$ & $2.50 \pm 1.22$ & 18.468 & $<0.00 I^{*}$ \\
\hline Nadir $\mathrm{SaO}_{2}(\%)$ & $66.4 \pm 12.56$ & $90.27 \pm 2.89$ & -12.422 & $<0.00 I^{*}$ \\
\hline Average $\mathrm{SaO}_{2}(\%)$ & $90.62 \pm 4.48$ & $95.53 \pm 2.41$ & -6.477 & $<0.00 I^{*}$ \\
\hline NI & $31.16 \pm 17.56$ & $10.09 \pm 3.66$ & 7.885 & $<0.00$ I* $^{*}$ \\
\hline N2 & $39.00 \pm 14.93$ & $40.7 I \pm 7.12$ & -0.693 & \\
\hline N3 & $22.96 \pm 18.14$ & $21.16 \pm 4.59$ & 0.645 & 0.520 \\
\hline REM & $7.04 \pm 7.87$ & $21.73 \pm 7.48$ & -9.076 & $<0.00 I^{*}$ \\
\hline $\mathrm{SaO}_{2}<90(\%)$ & $30.89 \pm 21.51$ & $0.354 \pm 0.20$ & 9.522 & $<0.00 I^{*}$ \\
\hline Arousal index/hour & $41.01 \pm 23.49$ & $11.96 \pm 2.75$ & 8.241 & $<0.00 I^{*}$ \\
\hline $\begin{array}{l}\text { Oxygen } \\
\text { desaturation index }\end{array}$ & $55.38 \pm 24.94$ & $2.95 \pm 1.28$ & 14.083 & $<0.00 I^{*}$ \\
\hline Sleep efficiency & $85.12 \pm 18.26$ & $92.24 \pm 6.24$ & -2.613 & $0.011 *$ \\
\hline MoCA, score & $25.18 \pm 2.13$ & $27.73 \pm 1.40$ & -6.708 & $<0.00 I^{*}$ \\
\hline ESS, score & $12.27 \pm 3.73$ & $4.22 \pm 1.55$ & 13.372 & $<0.00 I^{*}$ \\
\hline
\end{tabular}

Note: $* P<0.05$, which was considered statistically significant.

Abbreviations: OSA, obstructive sleep apnea; GSs, good sleepers; BMI, body mass index; $\mathrm{AHI}$, apnea-hypopnea index; REM, rapid eye movement; $\mathrm{SaO}_{2}<90 \%$, percentage of total sleep time spent at an oxygen saturation less than $90 \%$; MoCA, Montreal Cognitive Assessment; ESS, Epworth Sleepiness Scale; n, numbers.

which suggests that both the OSA patients and the GSs had typical features of small-world attributes in brain functional networks and that, compared with matched random networks, the brain functional networks exhibited much larger clustering coefficients and approximately equal shortest path lengths.

\section{OSA-related alterations in global network measures}

Despite the regime of small-worldness in both groups, statistical analyses revealed significant differences between the patients with OSA and the GSs in both small-world parameters and network efficiency. Using the AUC of each metric for statistical comparisons with the age, educational level, and mean FD as covariates, compared with the GS group, we found that the OSA group exhibited increased $\mathrm{Lp}(\mathrm{t}=2.789$, $P=0.006), \lambda(\mathrm{t}=2.920, P=0.004)$, and decreased $\gamma(\mathrm{t}=-2.899$, $P=0.005), \sigma(\mathrm{t}=-3.043, P=0.003)$. The network efficiency significantly decreased in $\mathrm{E}_{\text {glob }}(\mathrm{t}=-2.513, P=0.014)$. No significant differences were identified in the $\mathrm{Cp}(\mathrm{t}=1.752$, $P=0.083)$ or $\mathrm{E}_{\text {loc }}(\mathrm{t}=0.005, P=0.996)$. These results suggest that the small-world architecture and network efficiency were significantly altered in the brain functional networks of the OSA patients (Figure 1). 

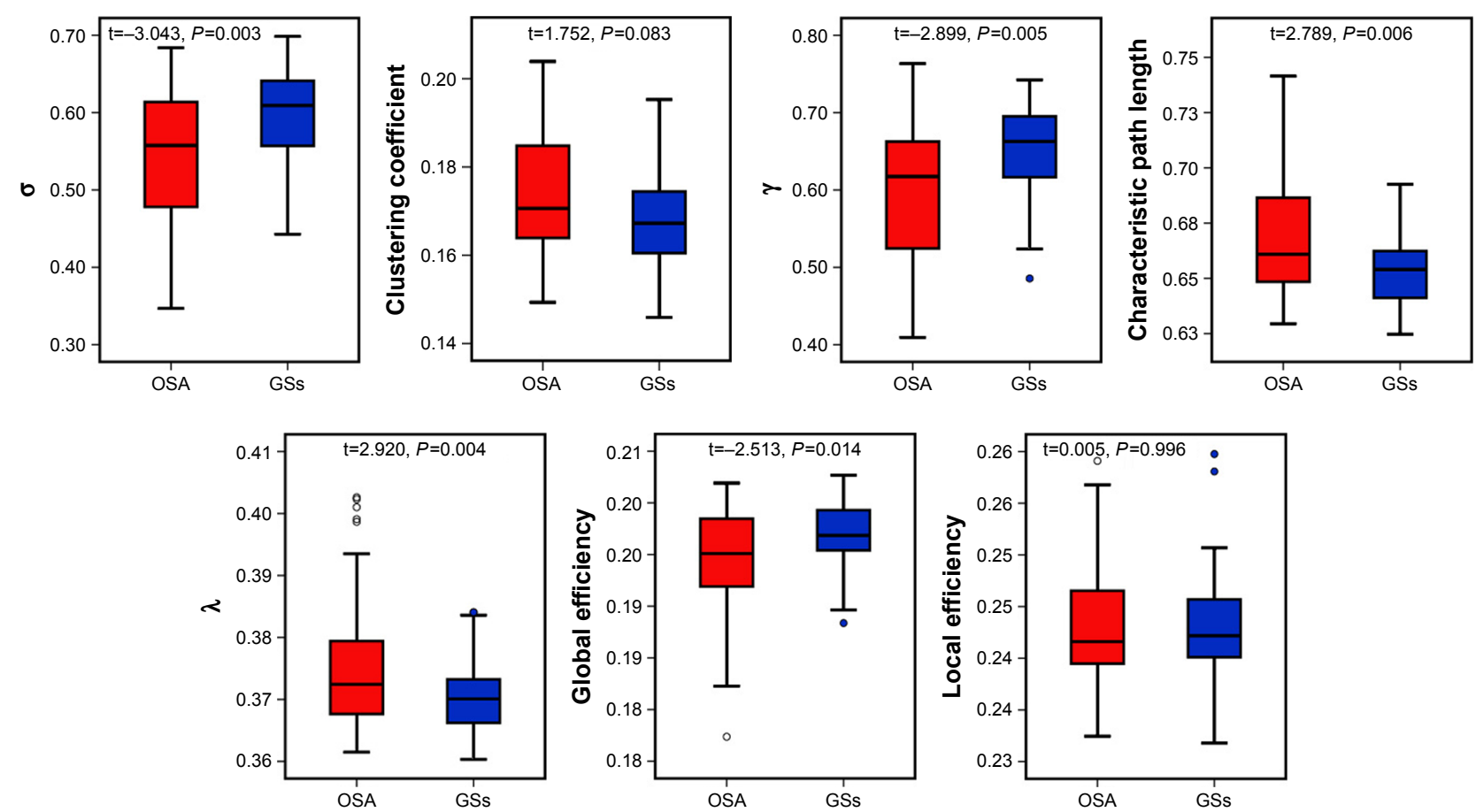

Figure I The small-world parameters and network efficiency parameters comparisons in patients with OSA and GSs.

Notes: Both the OSA and GSs exhibited small-world attribute. Compared to GSs, patients with OSA exhibited increased Lp, $\lambda$, and decreased $\gamma$, $\sigma$, and $\mathrm{E}_{\text {glob }}$ Abbreviations: $\sigma$, small-worldness; $\gamma$, normalized clustering coefficient; $\lambda$, normalized characteristic path length; OSA, obstructive sleep apnea; GSs, good sleepers.

\section{Nodal characteristics}

Regions that exhibited significant between-group differences in at least one of the three nodal centralities were considered abnormal nodal centralities in patients with OSA. The FDR correction was applied to each nodal characteristic, and none survived the correction for nodal betweenness $(P<0.01)$. Compared with GSs, the patients with OSA exhibited increased nodal centralities in the following brain regions: the left insula (INS), left heschl gyrus (HES), left cerebellum crus I, left cerebellum VIII, vermis VII, and vermis VIII. Decreased nodal centralities were found in the bilateral PCC, bilateral inferior parietal, but supramarginal and angular gyri (IPL), right inferior frontal gyrus, opercular part (IFGoperc), bilateral middle frontal gyrus, orbital part (ORBmid), and left angular gyrus (ANG) (Table 2).

\section{OSA-related alterations in FC}

Using the NBS approach, we identified a disconnected functional subnetwork with 14 nodes and 17 connections that was significantly altered in the OSA group (FDR-corrected $\mathrm{q}=0.01$ ). The disconnected functional subnetwork comprising pairs of nodes exhibited between-group differences in at least one of the three nodal centralities in the OSA group, and the nodes were mainly located in the prefrontal, parietal, and subcortical regions, as well as the cerebellum, which included several DMN regions (eg, the PCC, middle frontal gyrus, ANG), SN regions (eg, the insula, cerebellar vermis, and orbitofrontal gyrus), and CEN regions (eg, inferior frontal gyrus and inferior parietal lobe). The connections were mainly involved in long-distance connections linking different regions, including decreased FC in the prefrontalcerebellum and prefrontal-parietal, and increased FC in the parietal-cerebellum and parietal-prefrontal; connections were also involved in increased short-distance connections within parietal regions and cerebellum regions (Table 3 and Figure 2). Within this subnetwork, the mean connectivity values were not significantly different between the OSA group and the GSs ( $\mathrm{t}=0.355, P=0.723)$. The mean FC values showed significant positive correlation with global network metrics $\sigma(\mathrm{r}=0.329, P=0.029)$ and $\gamma(\mathrm{r}=0.385$, $P=0.011$; Figure 3).

\section{Relationships between network metrics and cognitive and clinical variables}

$\mathrm{E}_{\text {loc }}$ was positively correlated with the MoCA score ( $\mathrm{r}=0.298$, $P=0.049) . \mathrm{Cp}(\mathrm{r}=-0.381, P=0.011), \mathrm{Lp}(\mathrm{r}=-0.378, P=0.011)$, and $\lambda(\mathrm{r}=-0.378, P=0.012)$ were negatively correlated with the ESS score. $\sigma(\mathrm{r}=0.304, P=0.045)$ and $\mathrm{E}_{\text {glob }}(\mathrm{r}=0.325, P=0.031)$ were positively correlated with the oxygen desaturation index. 
Table 2 Between-group differences in nodal characteristics in patients with OSA and GSs

\begin{tabular}{|c|c|c|c|c|c|}
\hline \multirow[t]{2}{*}{ Brain region } & \multirow[t]{2}{*}{ Abbreviation } & \multicolumn{2}{|c|}{ Nodal degree } & \multicolumn{2}{|c|}{ Nodal efficiency } \\
\hline & & t-value & $P$-value & t-value & $P$-value \\
\hline \multicolumn{6}{|l|}{ OSA $>$ GSs } \\
\hline Left insula & INS.L & 2.710 & 0.008 & 2.185 & 0.031 \\
\hline Left heschl gyrus & HES.L & 3.174 & 0.002 & 2.693 & 0.008 \\
\hline Left cerebellum crus I & CRBLCrus-I.L & 3.326 & 0.001 & 2.734 & 0.008 \\
\hline Left cerebellum VIII & CRBL-VIIII.L & 4.119 & $<0.001$ & 3.535 & 0.001 \\
\hline Vermis VII & Vermis-VII & 2.851 & 0.005 & 2.443 & 0.017 \\
\hline Vermis VIII & Vermis-VIII & 3.089 & 0.003 & 2.525 & 0.013 \\
\hline \multicolumn{6}{|l|}{ OSA $<$ GSs } \\
\hline Left posterior cingulate cortex & PCC.L & -2.511 & 0.014 & -2.936 & 0.004 \\
\hline Right posterior cingulate cortex & PCC.R & -2.620 & 0.010 & -3.019 & 0.003 \\
\hline Left inferior parietal, but supramarginal and angular gyri & IPL.L & -2.868 & 0.005 & -3.195 & 0.002 \\
\hline Right inferior parietal, but supramarginal and angular gyri & IPL.R & -3.463 & 0.001 & -3.928 & $<0.001$ \\
\hline Right inferior frontal gyrus, opercular part & IFGoperc.R & -3.992 & $<0.001$ & -4.250 & $<0.001$ \\
\hline Left middle frontal gyrus, orbital part & ORBmid.L & -2.318 & 0.023 & -2.986 & 0.004 \\
\hline Rightmiddle frontal gyrus, orbital part & ORBmid.R & -2.834 & 0.006 & -3.288 & 0.001 \\
\hline Left angular gyrus & ANG.L & -2.929 & 0.004 & -3.492 & 0.001 \\
\hline
\end{tabular}

Note: The FDR correction was applied to each nodal characteristic, the $P$-value thresholds for both nodal degree and efficiency were 0.01 , and none survived the correction for betweenness.

Abbreviations: OSA, obstructive sleep apnea; GSs, good sleepers; INS, insula; HES, heschl gyrus; CRBL, cerebellum; PCC, posterior cingulate cortex; IPL, inferior parietal, but supramarginal and angular gyri; IFGoperc, inferior frontal gyrus, opercular part; ORBmid, bilateral middle frontal gyrus, orbital part; ANG, angular gyrus; FDA, false discovery rate.

Lp $(\mathrm{r}=-0.358, P=0.017)$ and $\lambda(\mathrm{r}=-0.368, P=0.014)$ were negatively correlated with the oxygen desaturation index.

\section{Discussion}

The present study applied graph-based theoretical approaches to analyze the topological organization of brain functional networks in patients with OSA. The results revealed that both the patients with OSA and the GSs had typical features of small-world attributes in brain functional networks. There were five main findings: (1) the patients with OSA exhibited decreased $\sigma, \gamma$, and $\mathrm{E}_{\text {glob }}$, and increased $\mathrm{Lp}$ and $\lambda$ in brain functional networks; (2) the patients with OSA exhibited increased nodal centralities in some $\mathrm{SN}$ regions, and decreased nodal centralities in some DMN, SN, and

Table 3 OSA-related alterations in FC

\begin{tabular}{|c|c|c|c|c|c|}
\hline Region I & Category & Region 2 & Category & t-value & $P$-value \\
\hline PCC.L & Parietal & IFGoperc.R & Prefrontal & 2.59 & 0.01 \\
\hline PCC.R & Parietal & IFGoperc.R & Prefrontal & 2.27 & 0.03 \\
\hline PCC.L & Parietal & INS.L & Subcortical & 2.2 & 0.03 \\
\hline IPL.L & Parietal & PCC.R & Parietal & 2.47 & 0.02 \\
\hline IPL.L & Parietal & PCC.L & Parietal & 3.04 & $<0.001$ \\
\hline IPL.R & Parietal & PCC.L & Parietal & 2.84 & 0.01 \\
\hline ANG.L & Parietal & ORBmid.L & Prefrontal & -2.14 & 0.04 \\
\hline IPL.R & Parietal & ORBmid.R & Prefrontal & -2.35 & 0.02 \\
\hline CRBLCrus-I.L & Cerebellum & ORBmid.L & Prefrontal & -3.34 & $<0.001$ \\
\hline CRBLCrus-I.L & Cerebellum & ORBmid.R & Prefrontal & -3.43 & $<0.001$ \\
\hline CRBL-VIII.L & Cerebellum & CRBLCrus-I.L & Cerebellum & 2.18 & 0.03 \\
\hline Vermis-VII & Cerebellum & CRBL-VIII.L & Cerebellum & 3.02 & $<0.001$ \\
\hline Vermis-VII & Cerebellum & PCC.L & Parietal & 2.18 & 0.03 \\
\hline Vermis-VIII & Cerebellum & IPL.R & Parietal & 2.1 & 0.04 \\
\hline Vermis-VIII & Cerebellum & CRBLCrus-I.L & Cerebellum & 2.94 & $<0.001$ \\
\hline Vermis-VIII & Cerebellum & CRBL-VIII.L & Cerebellum & 2.51 & 0.01 \\
\hline Vermis-VIII & Cerebellum & Vermis-VIII & Cerebellum & 3.6 & $<0.001$ \\
\hline
\end{tabular}

Abbreviations: OSA, obstructive sleep apnea; FC, functional connectivity; PCC, posterior cingulate cortex; IFGoperc, inferior frontal gyrus, opercular part; INS, insula; IPL, inferior parietal, but supramarginal and angular gyri; ANG, angular gyrus; ORBmid, middle frontal gyrus, orbital part; CRBLCrus-I, cerebellum crus I; CRBL-VIII, cerebellar lobule VIII. 


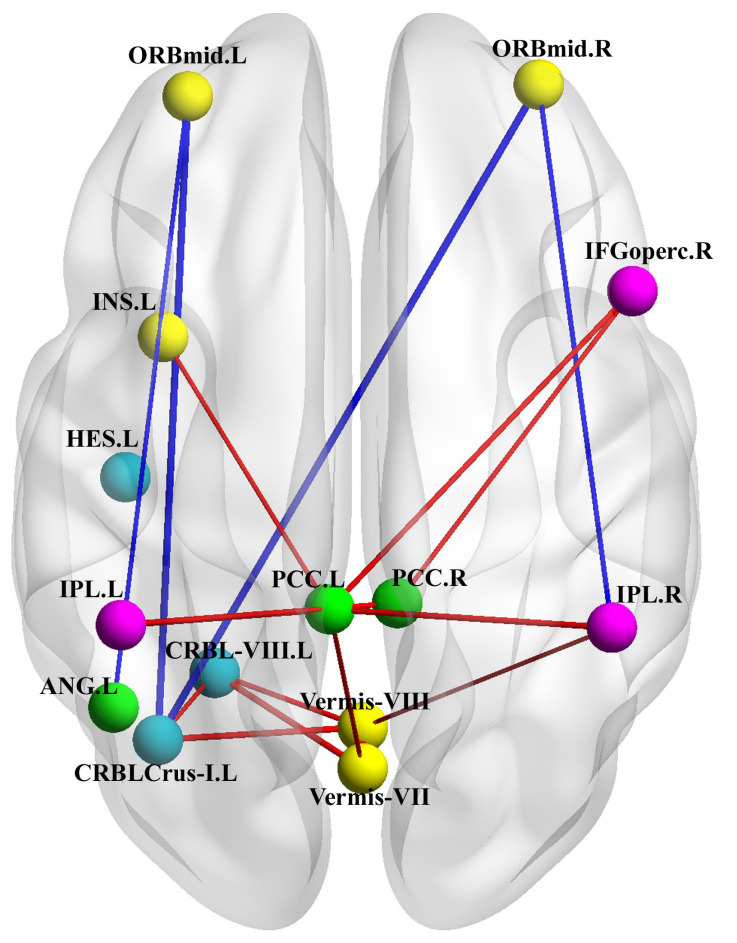

Default mode network

Salience network

Central executive network

Other

Figure 2 OSA-related alterations in FC.

Notes: A disconnected functional subnetwork with 14 nodes and 17 connections in the OSA group using a NBS approach (threshold, T=2.368, $P<0.01$ ). The OSA group exhibited abnormal long-distance and short-distance functional connections between/within DMN, SN, and CEN. The blue lines represent decreased rs-FC, and the red lines represent increased $r s-F C$ in patients with OSA compared to GSs. Abbreviations: OSA, obstructive sleep apnea; FC, functional connectivity; NBS, network-based statistics; DMN, default-mode network; SN, salience network; CEN, central executive network; rs-FC, resting state functional connectivity; GSs, good sleepers; ORBmid, middle frontal gyrus, orbital part; INS, insula; HES, heschl gyrus; IFGoperc, inferior frontal gyrus, opercular part; IPL, inferior parietal, but supramarginal and angular gyri; PCC, posterior cingulate cortex; CRBL-VIII, cerebellar lobule VIII; ANG, angular gyrus; CRBLCrus-I, cerebellum crus I.

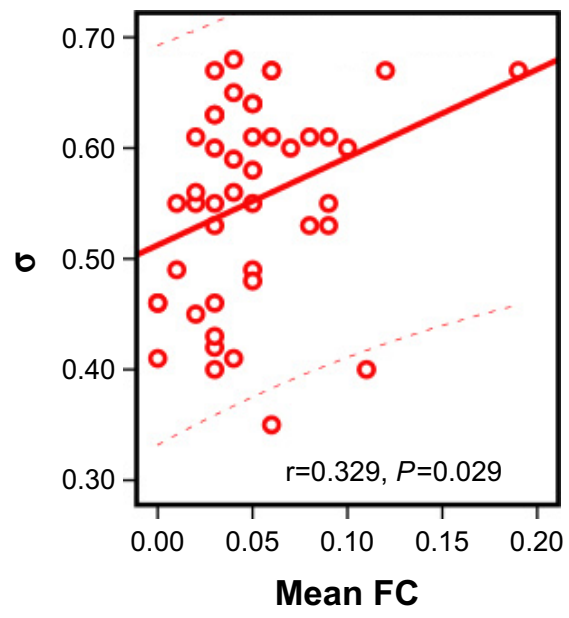

Figure 3 The relationship between the mean FC and global network metrics. Note: The mean functional connectivity was a positive correlation with $\sigma, \gamma$. Abbreviation: FC, functional connectivity.
CEN regions; (3) the patients with OSA exhibited abnormal long-distance and short-distance functional connections between/within DMN, SN, and CEN; (4) the mean FC value was significantly positively correlated with global network metrics $\sigma$ and $\gamma$; and (5) the global network metrics were significantly correlated with the ESS score, MoCA score, and oxygen desaturation index. Our findings indicate that individuals with OSA have disrupted global and regional properties and altered FC in brain functional networks, that the OSA-related altered FC further led to disrupted topological properties in brain functional networks, and that the disrupted topological properties are likely related to cognitive impairment, oxygen desaturation, and somnolence, thus providing a new insight into the understanding of the pathophysiology of OSA.

Both the patients with OSA and the GSs exhibited smallworld attributes in the brain functional networks, specifically a small-world network with both high clustering and short path lengths; these features provide a topological substrate that facilitates functional specialization and efficient integration and information transfer across the networks that are thought to constitute the basis of cognitive processes. Although both groups showed efficient economic small-world organization in their brain functional networks, the OSA group showed significantly increased $\mathrm{Lp}$, and $\lambda$ and decreased $\gamma, \mathrm{E}_{\text {glob }}$, and $\sigma$, which were consistent with the previous OSA-related brain network studies using structural MRI and fMRI, ${ }^{16,25,26}$ and the decreased $\sigma$ implies a disturbance in functional integration and segregation in the brain network. Lp minimizes the cost associated with routing action potentials across synaptic and axonal contacts, ensuring an inter-regional effective integrity or prompt information transfers across different nodes in the

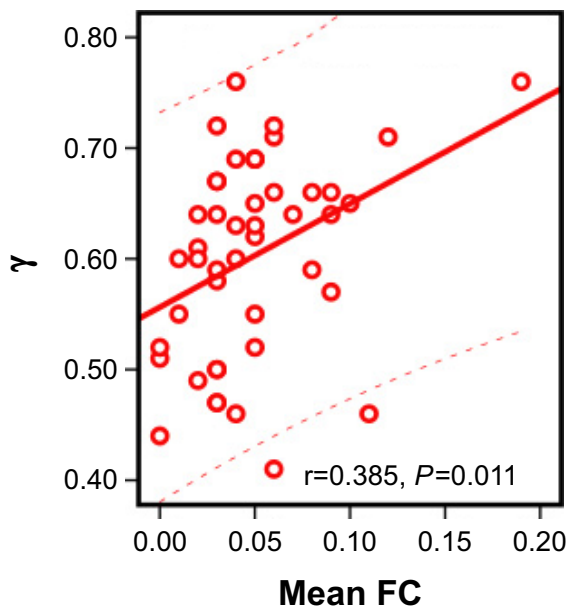


brain network. ${ }^{41}$ An increased $\lambda$ shows an increased ratio of characteristic shortest path lengths in the patients with OSA. The OSA-related increased Lp could be attributable to the degeneration of long-distance FC or sparse connectivity for information transmission. The clustering coefficient is often interpreted as an index of functional specialization or segregation or as a measure of the local information-processing capacity of a network. The present results also showed that the OSA patients had a decreased $\gamma$, which is consistent with previous findings. ${ }^{25}$ Our NBS results showed compensatory increased short-distance connections within parietal regions and cerebellar regions. The decreased $\gamma$ in the OSA patients suggests that the high functional specialization and segregation of the brain functional networks may be damaged, and decreased $\gamma$ might be due to the altered short-distance connections between adjacent brain regions in the patients with OSA. We also found that $\sigma$ was positively correlated with the oxygen desaturation index, $\mathrm{Cp}, \mathrm{Lp}$, and $\lambda$ were negatively correlated with the ESS score, and that Lp and $\lambda$ were negatively correlated with the oxygen desaturation index, which might provide clues to support that chronic intermittent hypoxemia disrupted the efficient integration and specialization of functional brain networks in OSA, and further resulted in cognitive impairment and daytime sleepiness.

Global efficiency, $\mathrm{E}_{\text {glob }}$, as a superior measure of integration, reflects information transfer between remote cortical regions across whole-brain regions, which are associated with long-range connections. ${ }^{25}$ In addition, our NBS results found that disrupted long-distance connections including decreased FC in the prefrontal-cerebellum and prefrontalparietal and increased FC in the parietal-prefrontal and parietal-cerebellum. Our finding of decreased $\mathrm{E}_{\text {glob }}$ suggests that the capacity for information exchange or functional integration of the brain networks in patients with OSA have been disrupted, which may result from the damaged or sparse functional connections across the whole-brain network in patients with OSA. This study also found that $\mathrm{E}_{\mathrm{loc}}$ was positively correlated with the MoCA score and that $\mathrm{E}_{\text {glob }}$ was positively correlated with the oxygen desaturation index, indicating that chronic intermittent hypoxemia was related to a reduced efficiency of information dissemination and damaged capacity for information integration between remote brain regions, and that aberrant network efficiency was related to cognitive function impairment in patients with OSA.

Nodal centrality detects the importance of a region in network communication by measuring the information flow in the functional connectome. ${ }^{41}$ We combined the betweenness centrality, nodal efficiency, and nodal degree to explore the node attributes of the brain functional connectome in patients with OSA. We found that the patients with OSA exhibited increased nodal centralities in several regions related to the SN (left insula and cerebellar vermis) and decreased nodal centralities in some DMN (bilateral PCC, bilateral middle frontal gyrus, and left ANG), SN (bilateral orbitofrontal gyrus), and CEN regions (right inferior frontal gyrus and bilateral inferior parietal lobe). Such findings are comparable to previous studies showing that patients with OSA demonstrate altered white matter integrity, regional gray matter volume, and regional cerebral blood flow, as well as brain metabolic abnormalities, connectivity deficits, and regional spontaneous activity deficits in these regions, which largely overlap with the DMN, SN, and CEN and explain the observed affective, sensorimotor, and cognitive deficits of OSA patients. ${ }^{6,11,13,15,44,45}$ The PCC has strong reciprocal connections with other structural areas that are components of the DMN and plays a crucial role in attention processing, cognitive functions, personal significance, evoked emotions, and the collection and evaluation of information. ${ }^{46}$ In addition, as abnormal brain activation, decreased gray matter volume and white matter integrity in the PCC are correlated with intermittent hypoxia and cognitive dysfunction in OSA, $, 9,13$ conceivably, decreased nodal centralities of the bilateral PCC might be involved in the disruption of cognitive function and emotional processing in OSA patients. The insula is thought to be related to sensorimotor integration, cognitive control, and emotional modulation. ${ }^{47}$ Bilateral anterior insular neuronal damage, localized cortical thinning in the insula, and abnormal insular cortex metabolites have been shown to be correlated with disease severity and neuropsychological status in patients with OSA. ${ }^{45,48}$ Park et $\mathrm{a}^{49}$ found that patients with OSA show complex abnormal FC between the insular cortices and several brain regions regulating affective, cognitive functions, autonomic, and sensorimotor functions. ${ }^{49}$ A meta-analysis highlighted that the insular cortex is an important node in neurocognitive, somatosensory, affective, and perceptual deficits in patients with OSA. ${ }^{50}$ We found increased nodal centralities in the left insula of the SN, which might be related to altered processing of cognitive, emotional, and sensorimotor integration in patients of OSA. These previous findings suggest that structural and functional deficits exist in the DMN, SN, and CEN, and we speculate that the structural changes and functional alterations observed in patients with OSA in those regions may impair regional centrality of brain networks and further diminish the integrative and communicative hub role of the DMN, SN, and CEN in patients with OSA. 
Prilipko et $\mathrm{al}^{10}$ observed defective deactivation of the DMN in patients with OSA during tasks and proposed nocturnal desaturation and intermittent hypoxia as a major factor behind the observed abnormal FC and DMN dysfunction in patients with OSA. The DMN is critical for maintaining brain function in the resting state and contributes to attentional internal mentation and adaptive function, ${ }^{51}$ and decreased rs-FC within the DMN may be related to depression and anxiety in patients with OSA. ${ }^{6}$ The SN plays a crucial role in detecting and processing cognitive, attention, control, emotional, and homeostatic saliency events. The right AIns, as a core area of the SN, plays a key role in switching between the DMN and CEN. ${ }^{52}$ Our NBS results showed decreased FC between the left insula of the SN and the left PCC of the DMN and increased FC between the orbital part of the left middle frontal gyrus of the SN and the left ANG of the DMN, which indicates that the FC between the DMN and the $\mathrm{SN}$ is disturbed in patients with OSA. Moreover, Zhang et $\mathrm{al}^{15}$ demonstrated a disconnect between the right insular cortex of the SN and DMN, which may be related to deficits in cognitive control, such as working memory, attention, and depression in patients with OSA. These findings suggest that the functional disconnect between the SN and DMN might play a crucial role in cognitive deficits and affective disturbances in patients with OSA. The CEN is usually observed to be active in task-positive tasks, which are associated with working memory, as well as processes related to goal-directed behaviors, attention, response selection, and task management. ${ }^{52,53}$ Altered spontaneous activation of the CEN and deactivation of the DMN during working memory tasks and a functional disconnect between brain regions of the CEN and DMN have been shown in rs-fMRI, which may contribute to cognitive deficits in OSA patients. ${ }^{8,10,13,15,50}$ Our results showed that the altered functional connections predominantly linked the $\mathrm{CEN}$ and the $\mathrm{DMN}$, which indicates a functional disconnection between the CEN and the DMN in patients with OSA. Combined with previous findings, we speculate that the aberrant nodal centralities may be relevant to the disconnection between the CEN and DMN, which further results in cognitive deficits in OSA patients, such as impairments in attention and working memory.

The present study had several limitations. First, we only recruited severe male patients with OSA, thus lacking an investigation of OSA severity or sex-specific differences, which prevents us from generalizing these findings to the total population of patients with OSA. Second, the functional brain networks were constructed by the AAL atlas, which parcellates the whole brain into 116 brain regions at a coarsely anatomical level; a more precise parcellation strategy or a functional scale should be used to construct the brain networks in the future. Third, OSA is related to depression and anxiety, which may affect the intrinsic resting-state brain activity, but we did not conduct an affective evaluation. ${ }^{54}$ Finally, we only used the MoCA to evaluate the cognitive function of subjects; a more detailed neuropsychological questionnaire should be used to disclose more interesting data.

\section{Conclusion}

In this rs-fMRI study, we applied graph-based theoretical approaches to investigate the topological properties of the functional connectome in OSA patients. We found that, although the patients with OSA showed small-world networks, the functional connectome of the patients with OSA had impaired global and regional properties and functional disconnections of the DMN, SN, and CEN; the aberrant topological attributes may be associated with disrupted FC and clinical and cognitive variables. These topological abnormalities and functional disconnections might be the potential biomarkers of cognitive impairments in patients with OSA.

\section{Acknowledgments}

This work was supported by grants from the Natural Science Foundation of China (Grant No 81560285), the Natural Science Foundation of Jiangxi, China (Grant No 20171BAB205070, 20132BAB205100), the Education Department Foundation of Jiangxi, China (Grant No 700544006), the Graduate Innovation Foundation of Jiangxi, China (Grant No YC2016-S100), the Science and Technology Support Program of Jiangxi, China (Grant No 20132BBG70061, 20141BBG70026), and the Doctoral Project Startup Fund (Grant No 700544005).

\section{Disclosure}

The authors report no conflicts of interest in this work.

\section{References}

1. Lim DC, Pack AI. Obstructive sleep apnea and cognitive impairment: addressing the blood-brain barrier. Sleep Med Rev. 2014;18(1):35-48.

2. Peppard PE, Young T, Barnet JH, Palta M, Hagen EW, Hla KM. Increased prevalence of sleep-disordered breathing in adults. Am J Epidemiol. 2013; 177(9):1006-1014.

3. Décary A, Rouleau I, Montplaisir J. Cognitive deficits associated with sleep apnea syndrome: a proposed neuropsychological test battery. Sleep. 2000;23(3):369-381.

4. Kumar R, Pham TT, Macey PM, Woo MA, Yan-Go FL, Harper RM. Abnormal myelin and axonal integrity in recently diagnosed patients with obstructive sleep apnea. Sleep. 2014;37(4):723-732.

5. Joo EY, Tae WS, Han SJ, Cho JW, Hong SB. Reduced cerebral blood flow during wakefulness in obstructive sleep apnea-hypopnea syndrome. Sleep. 2007;30(11):1515-1520. 
6. Li HJ, Nie X, Gong HH, Zhang W, Nie S, Peng DC. Abnormal restingstate functional connectivity within the default mode network subregions in male patients with obstructive sleep apnea. Neuropsychiatr Dis Treat. 2016;12:203-212.

7. Li HJ, Dai XJ, Gong HH, Nie X, Zhang W, Peng DC. Aberrant spontaneous low-frequency brain activity in male patients with severe obstructive sleep apnea revealed by resting-state functional MRI. Neuropsychiatr Dis Treat. 2015;11:207-214.

8. Santarnecchi E, Sicilia I, Richiardi J, et al. Altered cortical and subcortical local coherence in obstructive sleep apnea: a functional magnetic resonance imaging study. J Sleep Res. 2013;22(3):337-347.

9. Peng DC, Dai XJ, Gong HH, Li HJ, Nie X, Zhang W. Altered intrinsic regional brain activity in male patients with severe obstructive sleep apnea: a resting-state functional magnetic resonance imaging study. Neuropsychiatr Dis Treat. 2014;10:1819-1826.

10. Prilipko O, Huynh N, Schwartz S, et al. Task positive and default mode networks during a parametric working memory task in obstructive sleep apnea patients and healthy controls. Sleep. 2011;34(3) 293-301

11. Khazaie H, Veronese M, Noori K, et al. Functional reorganization in obstructive sleep apnoea and insomnia: a systematic review of the resting-state fMRI. Neurosci Biobehav Rev. 2017;77:219-231.

12. Macey PM, Kumar R, Ogren JA, Woo MA, Harper RM. Global brain blood-oxygen level responses to autonomic challenges in obstructive sleep apnea. PLoS One. 2014;9(8):e105261.

13. Zhang Q, Wang D, Qin W, et al. Altered resting-state brain activity in obstructive sleep apnea. Sleep. 2013;36(5):651-659.

14. Liu F, Guo W, Liu L, et al. Abnormal amplitude low-frequency oscillations in medication-naive, first-episode patients with major depressive disorder: a resting-state fMRI study. J Affect Disord. 2013;146(3): 401-406.

15. Zhang Q, Qin W, He X, et al. Functional disconnection of the right anterior insula in obstructive sleep apnea. Sleep Med. 2015;16(9): 1062-1070.

16. Park B, Palomares JA, Woo MA, et al. Disrupted functional brain network organization in patients with obstructive sleep apnea. Brain Behav. 2016;6(3):e00441.

17. Watts DJ, Strogatz SH. Collective dynamics of 'small-world' networks. Nature. 1998;393(6684):440-442.

18. He Y, Chen ZJ, Evans AC. Small-world anatomical networks in the human brain revealed by cortical thickness from MRI. Cereb Cortex. 2007; 17(10):2407-2419

19. Zhang J, Wang J, Wu Q, et al. Disrupted brain connectivity networks in drug-naive, first-episode major depressive disorder. Biol Psychiatry 2011;70(4):334-342.

20. John M, Ikuta T, Ferbinteanu J. Graph analysis of structural brain networks in Alzheimer's disease: beyond small world properties. Brain Struct Funct. 2017;222(2):923-942.

21. Lei D, Li K, Li L, et al. Disrupted functional brain connectome in patients with posttraumatic stress disorder. Radiology. 2015;276(3):818-827.

22. Liu F, Zhuo C, Yu C. Altered cerebral blood flow covariance network in schizophrenia. Front Neurosci. 2016;10:308.

23. Xu Y, Qiu S, Wang J, et al. Disrupted topological properties of brain white matter networks in left temporal lobe epilepsy: a diffusion tensor imaging study. Neuroscience. 2014;279:155-167.

24. Suo X, Lei D, Li K, et al. Disrupted brain network topology in pediatric posttraumatic stress disorder: a resting-state fMRI study. Hum Brain Mapp. 2015;36(9):3677-3686.

25. Luo YG, Wang D, Liu K, et al. Brain structure network analysis in patients with obstructive sleep apnea. PLoS One. 2015;10(9):e0139055.

26. Chen LT, Fan XL, Li HJ, et al. Disrupted small-world brain functional network topology in male patients with severe obstructive sleep apnea revealed by resting-state fMRI. Neuropsychiatr Dis Treat. 2017;13: 1471-1482.

27. Berry RB, Budhiraja R, Gottlieb DJ, et al. Rules for scoring respiratory events in sleep: update of the 2007 AASM manual for the scoring of sleep and associated events. J Clin Sleep Med. 2012;8(5):597-619.
28. Billings ME, Rosen CL, Auckley D, et al. Psychometric performance and responsiveness of the functional outcomes of sleep questionnaire and sleep apnea quality of life instrument in a randomized trial: the HomePAP study. Sleep. 2014;37(12):2017-2024.

29. Chen $\mathrm{KL}, \mathrm{Xu} \mathrm{Y}$, Chu AQ, et al. Validation of the Chinese version of Montreal Cognitive Assessment basic for screening mild cognitive impairment. J Am Geriatr Soc. 2016;64(12):e285-e290.

30. Nasreddine ZS, Phillips NA, Bédirian V, et al. The Montreal Cognitive Assessment, MoCA: a brief screening tool for mild cognitive impairment. J Am Geriatr Soc. 2005;53(4):695-699.

31. Yan CG, Wang XD, Zuo XN, Zang YF. DPABI: data processing \& analysis for (resting-state) brain imaging. Neuroinformatics. 2016; 14(3):339-351.

32. Van Dijk KR, Sabuncu MR, Buckner RL. The influence of head motion on intrinsic functional connectivity MRI. Neuroimage. 2012;59(1): 431-438.

33. Yan CG, Cheung B, Kelly C, et al. A comprehensive assessment of regional variation in the impact of head micromovements on functional connectomics. Neuroimage. 2013;76(1):183-201.

34. Tzourio-Mazoyer N, Landeau B, Papathanassiou D, et al. Automated anatomical labeling of activations in SPM using a macroscopic anatomical parcellation of the MNI MRI single-subject brain. Neuroimage. 2002;15(1):273-289.

35. Liu F, Guo W, Fouche JP, et al. Multivariate classification of social anxiety disorder using whole brain functional connectivity. Brain Struct Funct. 2015;220(1):101-115.

36. Liu F, Wang Y, Li M, et al. Dynamic functional network connectivity in idiopathic generalized epilepsy with generalized tonic-lonic seizure. Hum Brain Mapp. 2017;38(2):957-973.

37. Wang J, Wang X, Xia M, Liao X, Evans A, He Y. Corrigendum: GRETNA: a graph theoretical network analysis toolbox for imaging connectomics. Front Hum Neurosci. 2015;9:458.

38. He Y, Dagher A, Chen Z, et al. Impaired small-world efficiency in structural cortical networks in multiple sclerosis associated with white matter lesion load. Brain. 2009;132(12):3366-3379.

39. Achard S, Bullmore E. Efficiency and cost of economical brain functional networks. PLoS Comput Biol. 2007;3(2):e17.

40. Wang J, Wang L, Zang Y, et al. Parcellation-dependent small-world brain functional networks: a resting-state fMRI study. Hum Brain Mapp. 2009;30(5):1511-1523

41. Rubinov M, Sporns O. Complex network measures of brain connectivity: uses and interpretations. Neuroimage. 2010;52(3):1059-1069.

42. Genovese CR, Lazar NA, Nichols T. Thresholding of statistical maps in functional neuroimaging using the false discovery rate. Neuroimage. 2002;15(4):870-878.

43. Zalesky A, Fornito A, Bullmore ET. Network-based statistic: identifying differences in brain networks. Neuroimage. 2010;53(4):1197-1207.

44. Nie S, Peng DC, Gong HH, Li HJ, Chen LT, Ye CL. Resting cerebral blood flow alteration in severe obstructive sleep apnoea: an arterial spin labelling perfusion fMRI study. Sleep Breath. 2017;21(2):487-495.

45. Joo EY, Jeon S, Kim ST, Lee JM, Hong SB. Localized cortical thinning in patients with obstructive sleep apnea syndrome. Sleep. 2013;36(8): 1153-1162.

46. Torta DM, Cauda F. Different functions in the cingulate cortex, a meta-analytic connectivity modeling study. Neuroimage. 2011;56(4): 2157-2172.

47. Menon V, Uddin LQ. Saliency, switching, attention and control: a network model of insula function. Brain Struct Funct. 2010;214(5-6):655-667.

48. Yadav SK, Kumar R, Macey PM, Woo MA, Yan-Go FL, Harper RM. Insular cortex metabolite changes in obstructive sleep apnea. Sleep. 2014; 37(5):951-958.

49. Park B, Palomares JA, Woo MA, et al. Aberrant insular functional network integrity in patients with obstructive sleep apnea. Sleep. 2016; 39(5):989-1000

50. Tahmasian M, Rosenzweig I, Eickhoff SB, et al. Structural and functional neural adaptations in obstructive sleep apnea: an activation likelihood estimation meta-analysis. Neurosci Biobehav Rev. 2016;65:142-156. 
51. Andrews-Hanna JR, Reidler JS, Sepulcre J, Poulin R, Buckner RL. Functional-anatomic fractionation of the brain's default network. Neuron. 2010;65(4):550-562.

52. Sridharan D, Levitin DJ, Menon V. A critical role for the right frontoinsular cortex in switching between central-executive and default-mode networks. Proc Natl Acad Sci U S A. 2008;105(34):12569-12574.
53. Menon V. Large-scale brain networks and psychopathology: a unifying triple network model. Trends Cogn Sci. 2011;15(10):483-506.

54. Macey PM, Woo MA, Kumar R, Cross RL, Harper RM. Relationship between obstructive sleep apnea severity and sleep, depression and anxiety symptoms in newly-diagnosed patients. PLoS One. 2010; 5(4):e10211.

\section{Publish your work in this journal}

Neuropsychiatric Disease and Treatment is an international, peerreviewed journal of clinical therapeutics and pharmacology focusing on concise rapid reporting of clinical or pre-clinical studies on a range of neuropsychiatric and neurological disorders. This journal is indexed on PubMed Central, the 'PsycINFO' database and CAS, and is the official journal of The International Neuropsychiatric Association (INA). The manuscript management system is completely online and includes a very quick and fair peer-review system, which is all easy to use. Visit http://www.dovepress.com/testimonials.php to read real quotes from published authors.

\footnotetext{
Submit your manuscript here: http://www.dovepress.com/neuropsychiatric-disease-and-treatment-journal
} 